\title{
One-dimensional bargaining with Markov recognition probabilities
}

Citation for published version (APA):

Herings, P. J. J., \& Predtetchinski, A. (2007). One-dimensional bargaining with Markov recognition probabilities. METEOR, Maastricht University School of Business and Economics. METEOR Research Memorandum No. 044 https://doi.org/10.26481/umamet.2007044

Document status and date:

Published: 01/01/2007

DOI:

10.26481/umamet.2007044

Document Version:

Publisher's PDF, also known as Version of record

\section{Please check the document version of this publication:}

- A submitted manuscript is the version of the article upon submission and before peer-review. There can be important differences between the submitted version and the official published version of record.

People interested in the research are advised to contact the author for the final version of the publication, or visit the DOI to the publisher's website.

- The final author version and the galley proof are versions of the publication after peer review.

- The final published version features the final layout of the paper including the volume, issue and page numbers.

Link to publication

\footnotetext{
General rights rights.

- You may freely distribute the URL identifying the publication in the public portal. please follow below link for the End User Agreement:

www.umlib.nl/taverne-license

Take down policy

If you believe that this document breaches copyright please contact us at:

repository@maastrichtuniversity.nl

providing details and we will investigate your claim.
}

Copyright and moral rights for the publications made accessible in the public portal are retained by the authors and/or other copyright owners and it is a condition of accessing publications that users recognise and abide by the legal requirements associated with these

- Users may download and print one copy of any publication from the public portal for the purpose of private study or research.

- You may not further distribute the material or use it for any profit-making activity or commercial gain

If the publication is distributed under the terms of Article $25 \mathrm{fa}$ of the Dutch Copyright Act, indicated by the "Taverne" license above, 
P. Jean-Jacques Herings, Arkadi Predtetchinski

One-dimensional Bargaining with Markov

Recognition Probabilities

$\mathrm{RM} / 07 / 044$

JEL code: C78

\section{METE@R}

Maastricht research school of Economics of TEchnology and ORganizations

Universiteit Maastricht

Faculty of Economics and Business Administration P.O. Box 616

NL - 6200 MD Maastricht

phone : ++31433883830

fax : :+31433884873 


\title{
One-dimensional Bargaining with Markov Recognition Probabilities
}

\author{
P. Jean-Jacques Herings* and Arkadi Predtetchinski ${ }^{\dagger}$
}

October 12, 2007

\begin{abstract}
We study a process of bargaining over social outcomes represented by points in the unit interval. The identity of the proposer is determined by a general Markov process and the acceptance of a proposal requires the approval of it by all the players. We show that for every value of the discount factor below one the subgame perfect equilibrium in stationary strategies is essentially unique and equal to what we call the bargaining equilibrium. We provide a general characterization of the bargaining equilibrium. We consider next the asymptotic behavior of the equilibrium proposals when the discount factor approaches one. We give a complete characterization of the limit of the equilibrium proposals. We show that the limit equilibrium proposals of all the players are the same if the proposer selection process satisfies an irreducibility condition, or more generally, has a unique absorbing set. In general, the limit equilibrium proposals depend on the partition of the set of players in absorbing sets and transient states of the proposer selection process. We fully characterize the limit equilibrium proposals as the unique generalized fixed point of a particular function. This function depends in a simple way on the stationary distribution related to the proposer selection process. We compare the proposal selected according to our bargaining model to the one corresponding to the median voter theorem.
\end{abstract}

JEL classification code: C78.

Keywords: One-dimensional bargaining, Subgame perfect equilibrium, Stationary strategies, Markov process.

*Department of Economics, Maastricht University, P.O. Box 616, 6200 MD Maastricht, The Netherlands. E-mail: P.Herings@algec.unimaas.nl . The author would like to thank the Netherlands Organisation for Scientific Research (NWO) for financial support.

†Department of Economics, Maastricht University, P.O. Box 616, 6200 MD Maastricht, The Netherlands. E-mail: A.Predtetchinski@algec.unimaas.nl . The author would like to thank the Netherlands Organisation for Scientific Research (NWO) for financial support. 


\section{Introduction}

We consider the situation where a group of players has to choose an alternative out of a set of alternatives represented by points in a one-dimensional space. This problem features prominently in the literature on collective decision making and typical examples concern the choice of the taxation level, the location of a facility, or the amount of money devoted to a particular investment opportunity. A famous solution within this setting is the median voter result (Black, 1958). This result establishes conditions under which the chosen alternative corresponds to the most preferred one by the player with median preferences.

As in Banks and Duggan (2000), Cho and Duggan (2003), Kalandrakis (2006b), and Cardona and Ponsaí (2007), we analyze the situation from a bargaining perspective. Problems in collective decision making are often solved by bargaining. Politicians bargain about public good levels, tax rates, and issues in the traditional left-right spectrum in political decision making. Within firms bargaining takes place by executives to choose among a variety of investment opportunities. Unions have to aggregate heterogeneous member preferences, which may involve bargaining, and next bargain with firms on terms of employment. Bargaining problems within households and committees often lead to a one-dimensional set of alternatives.

Banks and Duggan (2000) consider bargaining over a set of social outcomes equal to an arbitrary compact convex subset of a Euclidean space. They examine a bargaining protocol where the proposer is selected according to a time-invariant probability distribution and they consider a general voting rule that determines whether a particular proposal is accepted or not. They prove the existence of a subgame perfect equilibrium in stationary strategies.

We restrict attention to the case where the set of social outcomes is the unit interval and where acceptance of a proposal requires unanimity of all the players involved in the decision making process. The process by which the proposer is selected follows a general Markov process. The probability by which a certain player is selected as proposer is also referred to as the recognition probability. We would like to emphasize the importance of a general model for the proposer selection process. Romer and Rosenthal (1978) is among the most influential papers emphasizing the role of proposal making on the selected outcome. Kalandrakis (2006a) shows in a bargaining framework that the proposer selection process is more important than voting rights, impatience, or complex equilibrium strategies in explaining political power. Empirical support for this feature in the context of the allocation of transportation funds in the U.S. is provided by Knight (2005).

Two important special cases of our proposer selection model are the ones where the proposer is selected according to some time-invariant probability distribution as in Banks and Duggan (2000) and Kalandrakis (2006b), or where the proposer is chosen according to some deterministically rotating scheme as in Cardona and Ponsatí (2007). Also the alternating offer bargaining model in Rubinstein (1982) is an example of a deterministically rotating scheme and is a special case of our more general model. Merlo and Wilson (1995) have generalized the Rubinstein set-up substantially and allow for a proposer selected by 
a Markov process. But since they consider bargaining problems where the dimension of the bargaining space equals the number of players minus one, one-dimensional bargaining with more than two players is not covered by their analysis.

The bargaining process we study proceeds as follows. At the beginning of a period one player is determined to make a proposal by means of a Markov process. The players react to the proposal sequentially and can each either accept or reject a proposal. If all players accept, the proposal is implemented, the game ends, and each player receives the payoff of the chosen alternative. Otherwise, the next period begins.

We study subgame perfect equilibria in stationary strategies. In a stationary strategy, each player makes a unique history-independent proposal and has a unique proposerdependent acceptance set. The acceptance set specifies which proposals are accepted by a player. The intersection of all the individual acceptance sets is called the social acceptance set. Like the individual acceptance set, the social acceptance set is proposer-dependent, but otherwise history-independent.

We show that subgame perfect equilibria in stationary strategies are essentially unique. All equilibria have the same equilibrium proposals, the same equilibrium payoffs, and the same social acceptance sets. In equilibrium, proposals are immediately accepted, so in equilibrium no delay occurs. Any two subgame perfect equilibria can only differ with respect to the individual acceptance sets. The difference is only relevant off the equilibrium path and only occurs when a player is indifferent between accepting or rejecting a particular proposal. The unique subgame perfect equilibrium in stationary strategies where players accept if they are indifferent between rejecting and accepting is referred to as the bargaining equilibrium.

Our uniqueness result complements the uniqueness results by Cho and Duggan (2003) and Cardona and Ponsatí (2007) in related frameworks, and the uniqueness result in Merlo and Wilson (1998) for the extension of Rubinstein (1982) to the $n$-person case that involves a stochastic cake under a contraction condition. Eraslan and Merlo (2002) show for the latter model that uniqueness does not generally hold when approval of a proposal is not required to be unanimous. Eraslan (2002) obtains a uniqueness result in the legislative bargaining approach of Baron and Ferejohn (1989). Kalandrakis (2006b) establishes a number of determinacy results (the number of equilibria is finite, and under some assumptions odd) for a quite general bargaining model.

We continue by studying the asymptotic behavior of subgame perfect equilibrium proposals as the discount factor converges to one. As an illustration, consider the special case of time-invariant recognition probabilities. These recognition probabilities give rise to a cumulative distribution function on the set of alternatives, by assigning to each alternative the mass of players whose most preferred alternative is less than or equal to that alternative. We prove that in the limit the bargaining equilibrium proposals of all players converge to the same proposal, being the (generalized) fixed point of the function equal to one minus the cumulative distribution function.

For the general case, consider a sequence of discount factors converging to one. The induced sequence of bargaining equilibria converges to some limit, which we call the limit equilibrium. It is not necessarily the case that limit equilibrium proposals of players are 
all the same. The configuration of limit equilibrium proposals depends on the partition of the set of players into absorbing sets and transient states, based on the proposer selection process. Starting from any transient state, the proposer selection process eventually enters one of the absorbing sets. The probability to enter a given absorbing set starting from a given transient state is called the absorption probability. The proposer selection process determines a unique stationary distribution on each absorbing set. This stationary distribution determines a generalized fixed point in a similar way as illustrated for the case of time-invariant recognition probabilities.

The limit equilibrium proposals can therefore be described as follows.

(A) Players in the same absorbing set make the same proposal.

(B) The proposal of a player in an absorbing set is the related generalized fixed point.

(C) The proposal of a player in a transient state is the weighted average of the absorbing set proposals, with weights given by the absorption probabilities.

It follows from Conditions (A) and (C) above that if there is only one absorbing set, then all players make the same limit equilibrium proposal. In particular, if the transition matrix of the proposer selection process is irreducible, the only absorbing set is the entire set of players. Also, if the recognition probabilities are time-invariant, the only absorbing set is the set of players with strictly positive recognition probabilities. Condition (B) provides a simple procedure to calculate limit equilibrium proposals. As a particular illustration, we show how the limit equilibrium compares to the median voter theorem, and we argue that it selects less extreme alternatives.

The paper has been organized as follows. Section 2 introduces the model and the notion of bargaining equilibrium. In Section 3 we show that each bargaining equilibrium is a subgame perfect equilibrium in stationary strategies, and conversely that each subgame perfect equilibrium in stationary strategies is essentially a bargaining equilibrium. In Section 4 we characterize bargaining equilibria by means of a specific system of equations and prove that for each value of the discount factor below one there is a unique bargaining equilibrium. Section 5 analyzes bargaining equilibria in a number of special cases, including the case of time-invariant recognition probabilities and a deterministically rotating scheme of proposers. Section 6 presents two results on stochastic matrices that are needed to show the main result of the paper, presented in Section 7. It fully characterizes the limit equilibrium and its relation to the generalized fixed point of the function generated by the stationary distribution of the proposer selection process. Section 8 concludes the paper.

\section{Bargaining Equilibrium}

We study an environment where the set of available alternatives or social states is represented by the unit interval. A finite set of players $N$ has to choose one alternative from the set $Z=[0,1]$. The implementation of an alternative $z \in[0,1]$ leads to instantaneous payoffs of $u_{i}(z)=1-\left|z-p_{i}\right|$ to player $i$. Thus $p_{i}$ is the ideal point of player $i$, and player 
$i$ 's payoff decreases linearly with the distance between the ideal point and the alternative. We assume that there are players for which 0 , respectively 1 , are the ideal points. We use the notation $i_{0}$ for a player with $p_{i_{0}}=0$ and $i_{1}$ for a player with $p_{i_{1}}=1$.

Given a discount factor $\delta \in[0,1)$, we define the bargaining game $\Gamma=\Gamma(\delta)$ as follows. The game $\Gamma$ is a dynamic game of perfect information. The game starts in period $t=0$. In each period $t$ nature selects a player to make a proposal and the selected player proposes one of the alternatives from $Z$. Then all players respond to the proposal. Each player can either reject or accept it. The players respond to a proposal sequentially, the order of the responses being history-independent. For simplicity, we assume that all players respond to the proposal, including the proposer himself. If the responders unanimously agree to a proposal, the game ends and the proposal is implemented. As soon as the first rejection occurs, time period $t+1$ begins.

If an alternative $z$ has been implemented in period $t$, the payoff to player $i$ is $\delta^{t} u_{i}(z)$. The payoff of perpetual disagreement is zero for every player.

The selection of proposers is determined by a Markov process on $N$. The transition probability from state $k$ to state $i$ is $\pi(i \mid k)$. When player $k$ makes a proposal in period $t$, then with probability $\pi(i \mid k)$ player $i$ will be selected to make a proposal in period $t+1$. Transition probabilities are collected in the matrix $\Pi$, with $\pi(i \mid k)$ in row $k$ and column $i$ of $\Pi$. In period $t=0$ the process is initialized by an arbitrary probability distribution on the set $N$.

Two important special cases of the proposer selection process are time-invariant recognition probabilities and deterministic recognition rules. Recognition probabilities are timeinvariant if there is a probability distribution $\mu$ on $N$ such that $\pi(i \mid k)=\mu(i)$ for all $(i, k) \in N \times N$. Recognition is deterministic if there is a function $r: N \rightarrow N$ such that $\pi(i \mid k)$ equals one if $i=r(k)$ and zero otherwise. The function $r$ is called a recognition rule. When $N$ consists of players $i_{0}$ and $i_{1}$ only and recognition is deterministic with the rule $r\left(i_{0}\right)=i_{1}$ and $r\left(i_{1}\right)=i_{0}$, we obtain the famous model of alternating offers bargaining of Rubinstein (1982) as a special case.

We will analyze subgame perfect equilibria in stationary strategies. In a stationary strategy a player chooses the same action at information sets with an identical continuation game. A stationary strategy of player $i$ consists of a proposal $x_{i} \in[0,1]$ and a collection $A_{i}$ of acceptance sets $A_{i \mid k} \subset[0,1]$ for $k \in N$. The set $A_{i \mid k}$ is the set of proposals of player $k$ that are accepted by player $i$. The stationary strategy $\left(x_{i}, A_{i}\right)$ therefore determines a unique behavioral strategy $\sigma_{i}$. A stationary strategy profile is a pair $(x, A)$, where $x=\left(x_{i}\right)_{i \in N}$ and $A=\left(A_{i}\right)_{i \in N}$.

A tuple of acceptance sets $A$ induces the social acceptance set $X_{k}=\cap_{i \in N} A_{i \mid k}$, i.e. the set of proposals by player $k$ that are unanimously accepted. We write $X=\left(X_{k}\right)_{k \in N}$.

Consider the case where all players make proposals that belong to the respective social acceptance set. Then there is no delay before a proposal is accepted. Such a strategy profile $(x, A)$ is called a no-delay strategy profile. A no-delay strategy profile $(x, A)$ induces a 
matrix of continuation payoffs $Y$ with element $y_{i \mid k}$ in row $k$ and column $i$ defined by

$$
y_{i \mid k}=\sum_{j \in N} \pi(j \mid k) u_{i}\left(x_{j}\right)
$$

The continuation payoff $y_{i \mid k}$ is next period's expected instantaneous payoff to player $i$ when the proposal made by player $k$ is rejected. Player $i$ should reject a proposal $x_{k}$ by player $k$ if $u_{i}\left(x_{k}\right)<\delta y_{i \mid k}$. These considerations motivate the definition of a bargaining equilibrium below.

Definition 2.1 A stationary strategy profile $(x, A)$ is a bargaining equilibrium of $\Gamma$ if

$$
\begin{aligned}
x_{k} & =\arg \max _{z \in X_{k}} u_{k}(z) \text { for each } k \in N, \\
A_{i \mid k} & =\left\{z \in Z \mid u_{i}(z) \geq \delta y_{i \mid k}\right\} \text { for each }(i, k) \in N \times N, \text { where } \\
y_{i \mid k} & =\sum_{j \in N} \pi(j \mid k) u_{i}\left(x_{j}\right) \text { for each }(i, k) \in N \times N, \\
X_{k} & =\cap_{i \in N} A_{i \mid k} \text { for each } k \in N .
\end{aligned}
$$

In a bargaining equilibrium no delay ever occurs as all equilibrium proposals $x_{k}$ lie in the respective social acceptance sets $X_{k}$. The number $y_{i \mid k}$ is the equilibrium continuation payoff to player $i$ after a proposal of player $k$ has been rejected. Player $i$ accepts a proposal $x_{k}$ of player $k$ if and only if the instantaneous payoff of $x_{k}$ is at least as high as the discounted continuation payoff.

\section{Subgame Perfect Equilibria in Stationary Strategies}

In this section we show that a bargaining equilibrium is a subgame perfect equilibrium in stationary strategies. Moreover, we show the converse result that if $(x, A)$ is a subgame perfect equilibrium in stationary strategies, then there is a bargaining equilibrium where all equilibrium proposals, all social acceptance sets, and all continuation payoffs coincide with those induced by $(x, A)$. As a consequence of the results in this section, we may restrict our analysis of $\Gamma$ to a study of its bargaining equilibria.

Theorem 3.1 A bargaining equilibrium $\sigma=(x, A)$ of $\Gamma$ is a subgame perfect equilibrium in stationary strategies.

We prove Theorem 3.1 in two steps. The first step (Proposition 3.1) is to show that there are no profitable one-shot deviations from the strategy $\sigma$ at any node of the game $\Gamma$. A strategy $\bar{\sigma}_{i}$ of player $i$ is said to be a one-shot deviation from $\sigma$ at node $h$ if it coincides with the strategy $\sigma_{i}$ on all nodes but $h$. The second step establishes the one-shot deviation property for the game $\Gamma$. The property states that if there is a profitable deviation

from a joint strategy $\sigma$, then there exists a profitable one-shot deviation. Proposition 3.2 establishes the the one-shot deviation property for $\Gamma$. 
Proposition 3.1 Let $\sigma=(x, A)$ be a bargaining equilibrium. Then no player has a oneshot profitable deviation from $\sigma$ at any node of the game $\Gamma$.

Proof. Let $\bar{\sigma}_{i}$ be a one-shot deviation by player $i$ from $\sigma$ at node $h$.

Suppose player $i$ has to make a proposal at node $h$. Under strategy $\sigma_{i}$ player $i$ proposes an alternative $x_{i}$. By the definition of bargaining equilibrium, the proposal $x_{i}$ is an element of the social acceptance set $X_{i}$ for the proposals of player $i$. It is therefore accepted, leading to a payoff of $u_{i}\left(x_{i}\right)$ for player $i$. In particular, $x_{i}$ is an element of $A_{i \mid i}$, so that $u_{i}\left(x_{i}\right) \geq \delta y_{i \mid i}$.

Suppose under strategy $\bar{\sigma}_{i}$ player $i$ makes a proposal $z$. If $z$ is not an element of the social acceptance set $X_{i}$ for the proposals of player $i$, it will be rejected. Because $\bar{\sigma}_{i}$ coincides with the strategy $\sigma_{i}$ on all nodes following $h$, player $i$ will receive a payoff of $\delta y_{i \mid i}$. But since $u_{i}\left(x_{i}\right) \geq \delta y_{i \mid i}$, the strategy $\bar{\sigma}_{i}$ is not a profitable deviation from $\sigma$. If $z$ is an element of $X_{i}$, then $z$ it is accepted and player $i$ receives a payoff of $u_{i}(z)$. However, in this case $u_{i}(z) \leq u_{i}\left(x_{i}\right)$, because by the definition of bargaining equilibrium, $x_{i}$ maximizes the function $u_{i}$ on the set $X_{i}$. Again, the strategy $\bar{\sigma}_{i}$ is not a profitable deviation from $\sigma$.

Suppose that at node $h$ player $i$ has to react to a proposal $z$ of player $k$. Suppose $z$ is accepted by player $i$ under strategy $\sigma_{i}$ but rejected under strategy $\bar{\sigma}_{i}$. Then the strategy $\sigma_{i}$ leads to a payoff of either $u_{i}(z)$ or $\delta y_{i \mid k}$, depending on whether other players accept or reject $z$. Since $z$ is accepted by player $i$, it is an element of the set $A_{i \mid k}$, so $u_{i}(z) \geq \delta y_{i \mid k}$. Because $\bar{\sigma}_{i}$ coincides with the strategy $\sigma_{i}$ on all nodes following $h$, it leads to payoff of $\delta y_{i \mid k}$ for player $i$. We see that $\bar{\sigma}_{i}$ is not a profitable deviation from $\sigma$.

Suppose $z$ is rejected by player $i$ under strategy $\sigma_{i}$ but is accepted under strategy $\bar{\sigma}_{i}$. Then the strategy $\sigma_{i}$ leads to a payoff of $\delta y_{i \mid k}$. Since $z$ is rejected by player $i$, it is not an element of $A_{i \mid k}$, so $u_{i}(z)<\delta y_{i \mid k}$. The strategy $\bar{\sigma}_{i}$ leads to payoff of either $u_{i}(z)$ or $\delta y_{i \mid k}$, depending on whether $z$ is accepted or rejected by other players. Again, $\bar{\sigma}_{i}$ is not a profitable deviation from $\sigma$.

Proposition 3.2 Let $\sigma$ be a profile of strategies. If player $i$ has a profitable deviation from $\sigma$, then player $i$ has a profitable one-shot deviation from $\sigma$.

Proof. Given a node $h$ we let $t(h)$ denote the period node $h$ belongs to. Suppose player $i$ has a profitable deviation $\bar{\sigma}_{i}$ from $\sigma$ in the subgame $\Gamma(\bar{h})$ of the game $\Gamma$ which increases the subgame payoff by $\varepsilon>0$. First we show that there is some $r$ such that player $i$ has a profitable deviation in the subgame $\Gamma(\bar{h})$ that coincides with $\sigma_{i}$ on all nodes except those corresponding to the first $r$ periods of the subgame $\Gamma(\bar{h})$.

Since the payoff player $i$ can get in period $t$ is bounded by $\delta^{t}$, the subgame payoff for any strategy that agrees with $\bar{\sigma}_{i}$ on all nodes corresponding to the first $t$ periods of the subgame differs from the payoff of $\bar{\sigma}_{i}$ by at most $\delta^{t}$. Set $r=\ln (\varepsilon) / \ln (\delta)$ and define the strategy $\sigma_{i}^{r}$ as follows:

$$
\sigma_{i}^{r}(h)= \begin{cases}\bar{\sigma}_{i}(h), & \text { if } t(h) \leq r+t(\bar{h}) \\ \sigma_{i}(h), & \text { otherwise }\end{cases}
$$


By definition, $\sigma_{i}^{r}$ coincides with $\bar{\sigma}_{i}$ on all nodes corresponding to the first $r$ periods of the subgame $\Gamma(\bar{h})$. Therefore, the subgame payoff of $\sigma_{i}^{r}$ differs from the payoff of $\bar{\sigma}_{i}$ by $\delta^{r}=\varepsilon$ at most. It follows that $\sigma_{i}^{r}$ is a profitable deviation from $\sigma_{i}$ in the subgame $\Gamma(\bar{h})$.

Suppose there is a node $h$ in the subgame $\Gamma(\bar{h})$ with $t(h)=r+t(\bar{h})$ such that player $i$ has to act at $h$ and strategy $\sigma_{i}^{r}$ is a profitable deviation from $\sigma$ in the subsubgame $\Gamma(h)$. Then $\sigma_{i}^{r}$ is a profitable one-shot deviation at node $h$. This follows from the fact that each player acts at most once every period. Thus if node $h^{\prime}$ follows node $h$ and player $i$ has to act at $h^{\prime}$, then $t\left(h^{\prime}\right)>r+t(\bar{h})$, implying that $\sigma_{i}^{r}\left(h^{\prime}\right)=\sigma_{i}\left(h^{\prime}\right)$. In this case the argument is complete.

Suppose there is no node $h$ in the subgame $\Gamma(\bar{h})$ with $t(h)=r+t(\bar{h})$ such that player $i$ has to act at $h$ and strategy $\sigma_{i}^{r}$ is a profitable deviation in the subsubgame $\Gamma(h)$. In this case we define a new strategy for $i$ as follows:

$$
\sigma_{i}^{r-1}(h)= \begin{cases}\sigma_{i}^{r}(h), & \text { if } t(h) \leq r+t(\bar{h})-1 \\ \sigma_{i}(h), & \text { otherwise. }\end{cases}
$$

Then the payoff of $\sigma_{i}^{r-1}$ at any node of the subgame $\Gamma(\bar{h})$ is at least as high as the payoff of $\sigma_{i}^{r}$. In particular, $\sigma_{i}^{r-1}$ is a profitable deviation from $\sigma$ in the subgame $\Gamma(\bar{h})$. Iterating this argument, we find a one-shot profitable deviation for player $i$.

Let $\sigma=(x, A)$ be a stationary strategy profile in the game $\Gamma$. Let $N_{a}=\left\{k \in N \mid x_{k} \in\right.$ $\left.X_{k}\right\}$ denote the set of players whose proposal is accepted and $N_{r}=\left\{k \in N \mid x_{k} \notin X_{k}\right\}$ denote the set of players whose proposal is rejected. We now compute the matrix $Y$ of continuation payoffs associated with $\sigma$. The matrix $Y$ contains $y_{i \mid k}$ in row $k$ and column $i$. If nature chooses a proposer $k$ from the set $N_{a}$, the payoff to player $i$ is $u_{i}\left(x_{k}\right)$, while if the proposer is chosen from $N_{r}$, then the payoff to player $i$ is $\delta y_{i \mid k}$. We have thus a system of equations

$$
y_{i \mid k}=\sum_{j \in N_{a}} \pi(j \mid k) u_{i}\left(x_{k}\right)+\delta \sum_{j \in N_{r}} \pi(j \mid k) y_{i \mid j} \text { for all }(i, k) \in N \times N
$$

Given a subset $S$ of $N$ let $1_{S}$ be the vector in $\mathbb{R}^{N}$ with each coordinate $i \in S$ equal to 1 and each coordinate $i \in N \backslash S$ equal to 0 . Let $\Omega(S)$ be a square matrix with the entries indexed by elements of the set $N \times N$. The only non-zero entries of $\Omega(S)$ are diagonal entries corresponding to the elements of the set $S$. These entries are equal to one. The matrix $\Omega(N)$ is equal to the identity matrix and is also denoted by $I$. Let $u(x)$ denote the matrix with the element $u_{i}\left(x_{k}\right)$ in row $k$ and column $i$. Then the above system can be written in the vector-matrix form as

$$
Y=\Pi \Omega\left(N_{a}\right) u(x)+\delta \Pi \Omega\left(N_{r}\right) Y .
$$

We can now solve for the continuation payoffs:

$$
Y=\Lambda u(x) \text {, where } \Lambda=\left[I-\delta \Pi \Omega\left(N_{r}\right)\right]^{-1} \Pi \Omega\left(N_{a}\right) .
$$


The matrix $\left[I-\delta \Pi \Omega\left(N_{r}\right)\right]$ is invertible and has a non-negative inverse, because the spectral radius of $\delta \Pi \Omega\left(N_{r}\right)$ is at most $\delta<1$. The matrix $\Lambda$ is therefore non-negative. All its columns corresponding to players in $N_{r}$ are equal to zero. The matrix $\Lambda$ equals the matrix $\Pi$ if $N_{r}$ is empty and has all entries equal to zero if $N_{a}$ is empty.

Furthermore, the sum of the entries of the matrix $\Lambda$ in any given row is at most 1 , that is $\Lambda 1_{N} \leq 1_{N}$. To prove this inequality, consider the following chain of equations:

$$
\begin{aligned}
{\left[I-\delta \Pi \Omega\left(N_{r}\right)\right][I-\Lambda] 1_{N} } & =\left[I-\delta \Pi \Omega\left(N_{r}\right)-\Pi \Omega\left(N_{a}\right)\right] 1_{N} \\
& =\left[I-\delta \Pi \Omega\left(N_{r}\right)-\delta \Pi \Omega\left(N_{a}\right)-(1-\delta) \Pi \Omega\left(N_{a}\right)\right] 1_{N} \\
& =\left[I-\delta \Pi-(1-\delta) \Pi \Omega\left(N_{a}\right)\right] 1_{N} \\
& =1_{N}-\delta 1_{N}-(1-\delta) \Pi 1_{N_{a}} \\
& =(1-\delta)\left(1_{N}-\Pi 1_{N_{a}}\right) \\
& =(1-\delta)\left(\Pi 1_{N}-\Pi 1_{N_{a}}\right) \\
& =(1-\delta) \Pi 1_{N_{r}} .
\end{aligned}
$$

The equation in the third line follows from the fact that $\Omega\left(N_{a}\right)+\Omega\left(N_{r}\right)=I$. The equation in the fourth line uses that $\Pi 1_{N}=1_{N}$ and $\Omega(S) 1_{N}=1_{S}$. The equation in the sixth line again uses the fact that $\Pi 1_{N}=1_{N}$. The equation in the last line uses the equation $1_{N_{a}}+1_{N_{r}}=1_{N}$.

Premultiplying the obtained equality by the matrix $\left[I-\delta \Pi \Omega\left(N_{r}\right)\right]^{-1}$ yields

$$
1_{N}-\Lambda 1_{N}=(1-\delta)\left[I-\delta \Pi \Omega\left(N_{r}\right)\right]^{-1} \Pi 1_{N_{r}},
$$

which is non-negative because $\left[I-\delta \Pi \Omega\left(N_{r}\right)\right]^{-1}$ is non-negative. This proves that $\Lambda 1_{N} \leq$ $1_{N}$.

With $\lambda(j \mid k)$ denoting the entry in the row $k$ and column $j$ of the matrix $\Lambda$, we can write the continuation payoff as

$$
y_{i \mid k}=\sum_{j \in N_{a}} \lambda(j \mid k) u_{i}\left(x_{j}\right) .
$$

Theorem 3.2 Suppose $\delta \in[0,1)$. Let $\sigma=(x, A)$ be a subgame perfect equilibrium of the game $\Gamma$ with continuation payoffs $Y$ and social acceptance sets $X$. Then there exists a bargaining equilibrium $(x, B)$ with continuation payoffs $Y$ and social acceptance sets $X$.

Proof. Define the following sets:

$$
\begin{aligned}
& B_{i \mid k}=\left\{z \in Z \mid u_{i}(z) \geq \delta y_{i \mid k}\right\} \text { and } B_{k}=\cap_{i \in N} B_{i \mid k}, \\
& C_{i \mid k}=\left\{z \in Z \mid u_{i}(z)>\delta y_{i \mid k}\right\} \text { and } C_{k}=\cap_{i \in N} C_{i \mid k} .
\end{aligned}
$$

Step 1. We prove that $\operatorname{Int} B_{k}=C_{k} \subset X_{k} \subset B_{k}$ for each $k$.

To prove the first inclusion, observe that $\operatorname{Int} B_{k}=\cap_{i \in N} \operatorname{Int} B_{i \mid k}=\cap_{i \in N} C_{i \mid k}=C_{k}$. 
To prove the second inclusion let $z$ be an element of $C_{k}$. Suppose $z$ is not an element of $X_{k}$. Let $i$ be the last player in the response order such that $z$ is not an element of the individual acceptance set $A_{i \mid k}$.

Consider any node $h$ of the game $\Gamma$ where player $i$ has to react to the proposal $z$ of player $k$. If all players follow the profile of strategies $\sigma$, then $z$ is rejected with player $i$ being the last player in the response sequence to reject $z$. Thus under strategy $\sigma$ the payoff at node $h$ to player $i$ is $\delta y_{i \mid k}$.

Consider a strategy $\bar{\sigma}_{i}$ of player $i$ that coincides with $\sigma_{i}$ on all nodes of the game $\Gamma$ except node $h$ where it assigns that player $i$ accept the proposal $z$. Because all players following $i$ in the response sequence accept $z$, playing $\bar{\sigma}_{i}$ against $\sigma$ in the subgame that starts at node $h$ leads to a payoff $u_{i}(z)$ for player $i$. Since $z \in C_{i \mid k}$ we know that $u_{i}(z)>\delta y_{i \mid k}$. Thus $\bar{\sigma}_{i}$ is a profitable one-shot deviation from $\sigma$ at node $h$. This contradicts the hypothesis that $\sigma$ is a subgame perfect equilibrium.

To prove the third inclusion let $z$ be an element of $X_{k}$ and suppose $z$ is not an element of $B_{k}$. Let $i$ be any player such that $z$ is not an element of the set $B_{i \mid k}$. Then $u_{i}(z)<\delta y_{i \mid k}$.

Consider any node $h$ of the game $\Gamma$ where player $i$ has to react to the proposal $z$ of player $k$. If all players follow the profile of strategies $\sigma$, then $z$ is accepted, resulting in payoff $u_{i}(z)$ for player $i$. Consider a strategy $\bar{\sigma}_{i}$ for player $i$ that coincides with $\sigma_{i}$ on all nodes of the game $\Gamma$ except node $h$ where it assigns that player $i$ reject the proposal $z$. Playing $\bar{\sigma}_{i}$ against $\sigma$ results in a payoff of $\delta y_{i \mid k}$ for player $i$. Thus $\bar{\sigma}_{i}$ is a profitable one-shot deviation from $\sigma$ at node $h$. This contradiction proves the second inclusion.

Step 2. We prove that the set $C_{k}$ is non-empty for each $k$.

Fix some $k \in N$. Let $N^{0}=\left\{i \in N \mid y_{i \mid k}=0\right\}$ and $N^{+}=\left\{i \in N \mid y_{i \mid k}>0\right\}$. Since all utility functions $u_{i}$ are positive on the interior of the unit interval, we have the inclusion

$$
(0,1) \subset \bigcap_{i \in N^{0}} C_{i \mid k}
$$

Let $\lambda(N \mid k)$ denote the sum of all entries in row $k$ of $\Lambda$. Suppose first that $\lambda(N \mid k)=0$. Then row $k$ of $\Lambda$ is zero, and therefore $y_{i \mid k}=0$ for all $i \in N$. Thus $N^{0}=N$ and we have the inclusion $(0,1) \subset C_{k}$. In this case the claim is proven.

Suppose now that $\lambda(N \mid k)>0$. Define the element $z$ of $Z$ by

$$
z=\frac{\sum_{j \in N_{a}} \lambda(j \mid k) x_{j}}{\lambda(N \mid k)} .
$$

For each $i \in N^{+}$we then have the following inequalities:

$$
u_{i}(z) \geq \frac{\sum_{j \in N_{a}} \lambda(j \mid k) u_{i}\left(x_{j}\right)}{\lambda(N \mid k)}=\frac{y_{i \mid k}}{\lambda(N \mid k)} \geq y_{i \mid k}>\delta y_{i \mid k},
$$

where the first inequality follows from the concavity of $u_{i}$, the second inequality from the fact that $\lambda(N \mid k) \leq 1$, and the third inequality from the fact that $\delta<1$ and $y_{i \mid k}>0$. We have thus established the inclusion

$$
z \in \bigcap_{i \in N^{+}} C_{i \mid k}
$$


Each $C_{i \mid k}$ is an open subset of $Z$. Since the set $N^{+}$is finite, $\cap_{i \in N^{+}} C_{i \mid k}$ is also an open subset of $Z$. As we have just shown, it is a non-empty set. Being a non-empty open subset of $Z$, it has to have a non-empty intersection with the interior of $Z$. The result follows.

Step 3. We prove that $N_{a}=N$.

Suppose not. Take some $k$ in $N_{r}$. We show that player $k$ has a profitable one-shot deviation from $\sigma$ at any node $h$ where he has to make a proposal.

Under strategy $\sigma$, player $k$ makes a proposal $x_{k}$ that is rejected, resulting in a payoff of $\delta y_{k \mid k}$ for player $k$. Take an arbitrary point $z$ in $C_{k}$ and consider a strategy that agrees with $\sigma_{k}$ on all nodes of the game $\Gamma$ except node $h$, where it assigns that player $k$ make a proposal $z$. Since $z \in C_{k} \subset X_{k}$, the alternative $z$ is unanimously accepted, resulting in the payoff of $u_{k}(z)$. Since $z \in C_{k \mid k}$, we have the inequality $u_{k}(z)>\delta y_{k \mid k}$. Proposing $z$ at node $h$ is therefore a profitable one-shot deviation from $\sigma$.

Step 4. We show that $x_{k}=\arg \max _{z \in X_{k}} u_{k}(z)$ for all $k \in N$.

From Step 3 we know that $x_{k} \in X_{k}$ for all $k$. If $x_{k}$ does not maximize the function $u_{k}$ on the set $X_{k}$, there exists an alternative $z \in X_{k}$ such that $u_{k}(z)>u_{k}\left(x_{k}\right)$. But then player $k$ has a profitable one-shot deviation from $\sigma$ at any node $h$ where he has to make a proposal, namely to propose the alternative $z$. Since $z \in X_{k}$, the alternative $z$ is unanimously accepted, resulting in the payoff of $u_{k}(z)$. This contradicts the fact that $\sigma$ is a subgame perfect equilibrium.

Step 5. We show that $X_{k}=B_{k}$ for all $k \in N$.

We know from Step 1 that $\operatorname{Int} B_{k} \subset X_{k} \subset B_{k}$. Since $B_{k}$ is a convex set, the set $X_{k}$ is also convex and its closure equals $B_{k}$. It is therefore sufficient to show that both $a=\inf X_{k}$ and $b=\sup X_{k}$ are contained in $X_{k}$.

From Step 4 we know that $x_{i_{0}}$ maximizes the utility function $u_{i_{0}}$ of player $i_{0}$ on $X_{k}$, and $x_{i_{1}}$ maximizes the utility function $u_{i_{1}}$ of player $i_{1}$ on $X_{k}$. But $u_{i_{0}}$ is a decreasing function and $u_{i_{1}}$ is an increasing function. Thus, we must have that $x_{i_{0}}=a$ and $x_{i_{1}}=b$. This proves the claim.

Step 6. We prove that $(x, B)$ is a bargaining equilibrium with continuation payoffs $Y$ and social acceptance sets $X$.

Equations (2) and (4) hold by definition of $B_{i \mid k}$ and $B_{k}=X_{k}$. We know from Step 3 that $N_{a}=N$. Therefore, the matrix $\Lambda$ equals $\Pi$ and $Y=\Pi u(x)$, which is Equation (3). Step 4 yields Equation (1).

\section{A Characterization of Bargaining Equilibria}

In this section we show that each game $\Gamma$ has a unique bargaining equilibrium. Together with Theorems 3.1 and 3.2 of Section 3, this implies that all subgame perfect equilibria in stationary strategies have the same equilibrium proposals, equilibrium utilities, and social acceptance sets.

Herings and Predtetchinski (2007) analyze a one-dimensional bargaining model where players can be clustered in two coalitions, one group having $z=0$ as the most preferred 
point, the other $z=1$. In that special case, there is no need to rely on stationarity of strategies to obtain a similar uniqueness result as in this section, and an analysis of subgame perfect equilibria suffices to obtain the desired result. Within the more general setting of this paper, it is possible to construct examples with multiple subgame perfect equilibria, as is also common in the literature on the extension of the Rubinstein model to the $n$-player case, see Herrero (1985) and Haller (1986).

Consider a bargaining equilibrium $(x, A)$ with continuation payoffs $Y$ and social acceptance sets $X$. Since the utility functions $u_{i}$ are concave, all individual acceptance sets are closed intervals. We shall use the notation $\left[a_{i \mid k}^{-}, a_{i \mid k}^{+}\right]$to denote the individual acceptance set $A_{i \mid k}$ of player $i$ for the proposals of player $k$. The social acceptance set $X_{k}$ for the proposals of player $k$ is also a closed interval, denoted by $\left[x_{k}^{-}, x_{k}^{+}\right]$.

We now present the two main theorems of this section. Theorem 4.1 is a characterization of bargaining equilibria by means of a system of equations in terms of the equilibrium proposals and the social acceptance sets. Theorem 4.2 states that the bargaining equilibrium is unique.

The system (5)-(6) below is referred to as the characteristic system of equations.

$$
\begin{gathered}
x^{-}=\delta \Pi x, \quad x^{+}=(1-\delta) 1_{N}+\delta \Pi x, \\
x_{k}=\left\{\begin{array}{ll}
x_{k}^{-}, & \text {if } p_{k} \leq x_{k}^{-}, \\
p_{k}, & \text { if } x_{k}^{-} \leq p_{k} \leq x_{k}^{+}, \\
x_{k}^{+}, & \text {if } x_{k}^{+} \leq p_{k},
\end{array} \quad \text { for all } k \in N .\right.
\end{gathered}
$$

Theorem 4.1 Let $(x, A)$ be a bargaining equilibrium with social acceptance sets $X_{k}=$ $\left[x_{k}^{-}, x_{k}^{+}\right]$. Then the triple $\left(x, x^{-}, x^{+}\right)$is a solution to the characteristic system of equations. Conversely, suppose the triple $\left(x, x^{-}, x^{+}\right)$is a solution to the characteristic system of equations. Then there exists a bargaining equilibrium with $x$ the equilibrium proposal profile and $\left[x_{k}^{-}, x_{k}^{+}\right]$the social acceptance set for the proposals of player $k$.

Theorem 4.2 There exists a unique bargaining equilibrium.

The remainder of the section is devoted to the proof of these two results. If $x_{k}$ is an equilibrium proposal of player $k$ in a bargaining equilibrium, then it is the point of $X_{k}$ closest to the ideal point $p_{k}$, whence equation (6). To derive Equation (5) we rely on Propositions 4.1 and 4.2 below. Proposition 4.1 essentially says that the players whose ideal points are 0 and 1 determine all social acceptance sets. Player $i_{1}$ whose ideal point is 1 determines the lower endpoint of each social acceptance set, while player $i_{0}$ with ideal point is 0 determines the upper endpoint.

Proposition 4.1 Suppose the tuple (x, A,Y,X) satisfies Equations (2), (3), and (4). Let $A_{i \mid k}=\left[a_{i \mid k}^{-}, a_{i \mid k}^{+}\right]$and $X_{k}=\left[x_{k}^{-}, x_{k}^{+}\right]$. Then

(a) $x_{k}^{-}=\max _{i \in N}\left\{a_{i \mid k}^{-}\right\}$and $x_{k}^{+}=\min _{i \in N}\left\{a_{i \mid k}^{+}\right\}$, 
(b) if $p_{i} \leq p_{j}$, then $x_{i \mid k}^{-} \leq x_{j \mid k}^{-}$and $x_{i \mid k}^{+} \leq x_{j \mid k}^{+}$,

(c) the equations $x_{k}^{-}=a_{i_{1} \mid k}^{-}$and $x_{k}^{+}=a_{i_{0} \mid k}^{+}$hold, and

(d) $X_{k}=A_{i_{0} \mid k} \cap A_{i_{1} \mid k}$.

Proof. Claim (a) follows directly from Equation (4). To prove claim (b), notice that $a_{i \mid k}^{-}=\max \left\{0, z_{i \mid k}^{-}\right\}$and $a_{i \mid k}^{+}=\min \left\{1, z_{i \mid k}^{+}\right\}$, where

$$
z_{i \mid k}^{-}=p_{i}-\left(1-\delta y_{i \mid k}\right) \text { and } z_{i \mid k}^{+}=p_{i}+\left(1-\delta y_{i \mid k}\right) \text {. }
$$

As $\max \{0, \cdot\}$ and $\min \{1, \cdot\}$ are non-decreasing functions, it is sufficient to show that $z_{i \mid k}^{-} \leq$ $z_{j \mid k}^{-}$and $z_{i \mid k}^{+} \leq z_{j \mid k}^{+}$whenever $p_{i} \leq p_{j}$.

For all real $z$ and $\dot{z}$ the inequality $|z|-|\dot{z}| \leq|z-\dot{z}|$ holds. Consequently, for each $i$ and $j$ in $N$ and each $z$ in $Z$ we have $u_{i}(z)-u_{j}(z)=\left|p_{i}-z\right|-\left|p_{j}-z\right| \leq\left|p_{i}-p_{j}\right|$, so $\left|u_{i}(z)-u_{j}(z)\right| \leq\left|p_{i}-p_{j}\right|$. It follows that

$$
\left|y_{i \mid k}-y_{j \mid k}\right| \leq \sum_{l \in N} \pi(l \mid k)\left|u_{i}\left(x_{l}\right)-u_{j}\left(x_{l}\right)\right| \leq\left|p_{i}-p_{j}\right| .
$$

Now suppose that $p_{i} \leq p_{j}$. Then

$$
\begin{aligned}
& z_{i \mid k}^{-}-z_{j \mid k}^{-}=p_{i}-p_{j}+\delta\left(y_{i \mid k}-y_{j \mid k}\right) \leq p_{i}-p_{j}+\delta\left|p_{i}-p_{j}\right|=(1-\delta)\left(p_{i}-p_{j}\right) \leq 0 \\
& z_{i \mid k}^{+}-z_{j \mid k}^{+}=p_{i}-p_{j}-\delta\left(y_{i \mid k}-y_{j \mid k}\right) \leq p_{i}-p_{j}+\delta\left|p_{i}-p_{j}\right|=(1-\delta)\left(p_{i}-p_{j}\right) \leq 0 .
\end{aligned}
$$

Claim (c) follows immediately from (a) and (b). Claim (d) follows from (c) and from the fact that $A_{i_{0} \mid k}=\left[0, a_{i_{0} \mid k}^{+}\right]$and $A_{i_{1} \mid k}=\left[a_{i_{1} \mid k}^{-}, 1\right]$.

Proposition 4.2 Suppose the tuple (x, A,Y,X) satisfies Equations (2), (3), and (4). Let $X_{k}=\left[x_{k}^{-}, x_{k}^{+}\right]$. Then $x^{-}=\delta \Pi x$ and $x^{+}=(1-\delta) 1_{N}+\delta \Pi x$.

Proof. We already know from Proposition 4.1 that $x_{k}^{-}=a_{i_{1} \mid k}^{-}$and $x_{k}^{+}=a_{i_{0} \mid k}^{+}$. Now the utility function of player $i_{1}$ is $u_{i_{1}}(z)=z$. Thus $y_{i_{1} \mid k}=(\Pi x)_{k}$. It follows that $a_{i_{1} \mid k}^{-}=\delta(\Pi x)_{k}$. The utility function of player $i_{0}$ is $u_{i_{0}}(z)=1-z$. Therefore, $y_{i_{0} \mid k}=1-(\Pi x)_{k}$. It follows that $a_{i_{0} \mid k}^{+}=1-\delta+\delta(\Pi x)_{k}$. The result follows.

Now the proof of Theorem 4.1 is immediate. Suppose $(x, A)$ is a bargaining equilibrium with continuation payoffs $Y$ and social acceptance sets $X=\left(\left[x_{k}^{-}, x_{k}^{+}\right]\right)_{k \in N}$. Equation (6) then holds because $x_{k}$ is the point of the social acceptance set $\left[x_{k}^{-}, x_{k}^{+}\right]$closest to the point $k$. Equation (5) holds by Proposition (4.2). Conversely, suppose the triple $\left(x, x^{-}, x^{+}\right)$is a solution to the characteristic system of equations. Define $Y, A$, and $X$ by Equations (3), (2), and (4), respectively, and let $X_{k}=\left[\dot{x}_{k}^{-}, \dot{x}_{k}^{+}\right]$. We must show that Equation (1) holds and that $\dot{x}^{-}=x^{-}$and $\dot{x}^{+}=x^{+}$. Now, since the tuple the tuple $(x, A, Y, X)$ satisfies 
Equations (2), (3), and (4), Proposition 4.2 implies that $\dot{x}^{-}=\delta \Pi x$ and $\dot{x}^{+}=1-\delta+\delta x$. Equation (5) now implies that $\dot{x}^{-}=x^{-}$and $\dot{x}^{+}=x^{+}$. Equation (6) implies Equation (1). Thus $(x, A)$ is a bargaining equilibrium.

Now we turn to the proof of the uniqueness result. Given $i \in N$ and $z \in Z$, let $h_{i}(z)$ be the point of the interval $[\delta z, 1-\delta+\delta z]$ closest to the point $p_{i}$. Define the function $F: Z^{N} \rightarrow Z^{N}$ by letting $F_{i}(x)=h_{i}\left((\Pi x)_{i}\right)$ for each $x$ in $Z^{N}$. It follows from Theorem 4.1 that $x$ is an equilibrium proposal profile in a bargaining equilibrium if and only if $x$ is a fixed point of the function $F$. Proposition 4.3 below shows that the function $F$ is a contraction with respect to the norm on $Z^{N}$ given by $\|x\|=\max \left|x_{i}\right|$. It then follows that $F$ has a unique fixed point, thus establishing Theorem 4.2.

\section{Proposition 4.3}

(a) For each $i \in N$, each $z$ and $\dot{z}$ in $Z,\left|h_{i}(z)-h_{i}(\dot{z})\right| \leq \delta|z-\dot{z}|$.

(b) For each $x$ in $\mathbb{R}^{N},\|\Pi x\| \leq\|x\|$.

(c) For each $x$ and $\dot{x}$ in $Z^{N},\|F(x)-F(\dot{x})\| \leq \delta\|x-\dot{x}\|$.

Proof. To prove claim (a) write the function $h_{i}$ as

$$
h_{i}(z)= \begin{cases}1-\delta+\delta z, & \text { if } z \in Z_{1}=\left[0,\left(p_{i}+\delta-1\right) / \delta\right], \\ p_{i}, & \text { if } z \in Z_{2}=\left[\left(p_{i}+\delta-1\right) / \delta, p_{i} / \delta\right], \\ \delta z, & \text { if } z \in Z_{3}=\left[p_{i} / \delta, 1\right] .\end{cases}
$$

The function $h_{i}$ is affine with a slope of $\delta$ on $Z_{1}$ and $Z_{3}$ and it is a constant on $Z_{2}$. The result follows.

To prove claim (b), observe that for each $k \in N$ we have the inequalities

$$
\left|(\Pi x)_{k}\right|=\left|\sum_{i \in N} \pi(i \mid k) x_{i}\right| \leq \sum_{i \in N} \pi(i \mid k)\left|x_{i}\right| \leq \sum_{i \in N} \pi(i \mid k)\|x\|=\|x\| .
$$

The result follows.

To prove claim (c) we compute

$$
\begin{aligned}
\left|F_{i}(x)-F_{i}(\dot{x})\right| & =\left|h_{i}\left((\Pi x)_{i}\right)-h_{i}\left((\Pi \dot{x})_{i}\right)\right| \\
& \leq \delta\left|(\Pi x)_{i}-(\Pi \dot{x})_{i}\right| \\
& =\delta\left|(\Pi(x-\dot{x}))_{i}\right| \\
& \leq \delta\|x-\dot{x}\| .
\end{aligned}
$$

The result follows. 


\section{Some Special Cases}

Motivated by Theorem 4.1, we refer to the solution $\left(x, x^{-}, x^{+}\right)$of the system $5-6$ of characteristic equations as a bargaining equilibrium.

\subsection{Time-invariant recognition probabilities}

When the recognition probabilities are time-invariant, the continuation payoff $y_{i \mid k}$ of player $i$ does not depend on the identity of the proposer, $k$. In particular, a rejection of a proposal by player $k$ leads to the same continuation payoff as a rejection of a proposal by player $j$. It follows that the individual acceptance set $A_{i \mid k}$ of player $i$ for the proposals of player $k$ does not depend on $k$. Therefore, also the social acceptance sets $X_{k}$ do not depend on $k$ and are all equal to some interval $[a, b]$.

Let $\mu$ be time-invariant recognition probabilities. The characteristic system of equations then simplifies as follows:

$$
\begin{gathered}
a=\delta \mu x, \quad b=1-\delta+\delta \mu x, \\
x_{k}=\left\{\begin{array}{ll}
a, & \text { if } p_{k} \leq a \\
p_{k}, & \text { if } a \leq p_{k} \leq b \\
b, & \text { if } b \leq p_{k},
\end{array} \quad \text { for all } k \in N,\right.
\end{gathered}
$$

where $\mu x=\sum_{i \in N} \mu(i) x_{i}$. The equilibrium proposals are given by Figure 1 below. Notice that the equilibrium proposal $x_{i}$ is non-decreasing in $p_{i}$. An interesting feature of a bargaining equilibrium in the model with time-invariant recognition probabilities is that it is fully characterized by two numbers, namely $a$ and $b$, the endpoints of the common social acceptance set.

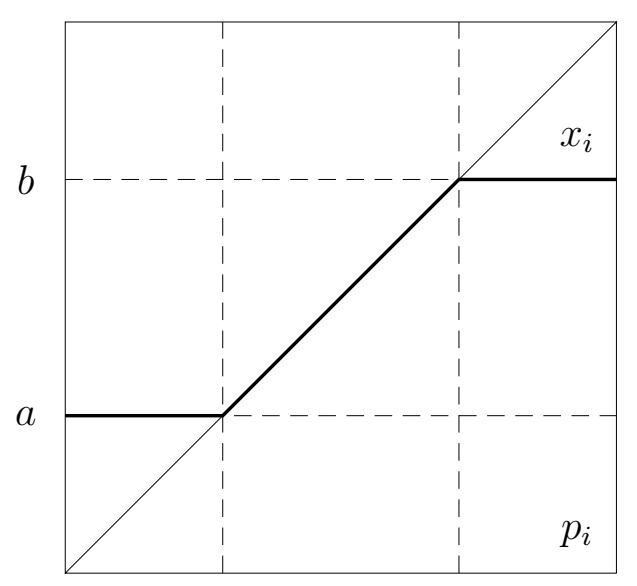

Figure 1: Equilibrium proposal $x_{i}$ in the case of time-invariant recognition probabilities.

Given a discount factor $\delta \in[0,1)$ we let $(x(\delta), a(\delta), b(\delta))$ denote the unique bargaining equilibrium in a model with time-invariant recognition probabilities $\mu$. The limit of the 
bargaining equilibrium as $\delta$ approaches one, if it exists, is called the limit equilibrium. Now we compute the limit equilibrium for the case of time-invariant recognition probabilities. In Section 7 we compute the limit equilibrium for the general case.

Definition 5.1 A point $z \in Z$ is said to be a generalized fixed point of a non-increasing function $f: \mathbb{R} \rightarrow Z$ if $f(z+\varepsilon) \leq z \leq f(z-\varepsilon)$ for all $\varepsilon>0$.

It is clear that each non-increasing function has at most one generalized fixed point.

Define a cumulative distribution function $F: \mathbb{R} \rightarrow Z$ by the equation

$$
F(z)=\mu\left(\left\{i \in N \mid p_{i} \leq z\right\}\right)
$$

Thus $F(z)$ is the mass of players whose ideal point lie in the interval $[0, z]$. It is clear that $F$ is a non-decreasing function. Theorem 5.1 characterizes limit equilibria as the generalized fixed point of the function $1-F$. The proof of the theorem relies on the following result.

Proposition 5.1 Let $(x, a, b)$ be a bargaining equilibrium in a model with time-invariant recognition probabilities. Then $1-F(b) \leq \mu x \leq 1-F(a)$.

Proof. Define the elements $z^{-}$and $z^{+}$of $Z^{N}$ as follows:

$$
z_{i}^{-}=\left\{\begin{array}{ll}
a, & \text { if } p_{i} \in[0, b], \\
b, & \text { otherwise }
\end{array} \quad \text { and } \quad z_{i}^{+}= \begin{cases}a, & \text { if } p_{i} \in[0, a] \\
b, & \text { otherwise }\end{cases}\right.
$$

It is obvious that $z^{-} \leq x \leq z^{+}$. Therefore $\mu z^{-} \leq \mu x \leq \mu z^{+}$. Now, we use Equation (7) to compute:

$$
\begin{aligned}
& \mu z^{-}=F(b) a+(1-F(b)) b=(1-\delta)(1-F(b))+\delta \mu x, \\
& \mu z^{+}=F(a) a+(1-F(a)) b=(1-\delta)(1-F(a))+\delta \mu x .
\end{aligned}
$$

This yields the desired inequalities $1-F(b) \leq \mu x \leq 1-F(a)$.

Theorem 5.1 The limits $\lim _{\delta \uparrow 1} x_{i}(\delta)$ for $i \in N, \lim _{\delta \uparrow 1} a(\delta)$, and $\lim _{\delta \uparrow 1} b(\delta)$ exist. All limits are equal to the unique generalized fixed point of the function $1-F$.

Proof. Let $\delta_{n}$ be a sequence in $[0,1)$ converging to 1 . We must show that the sequences $x_{i}\left(\delta_{n}\right)$ for each $i \in N, a\left(\delta_{n}\right)$ and $b\left(\delta_{n}\right)$ converge to the generalized fixed point of the function $1-F$.

Without loss of generality, assume that the sequences $x_{i}\left(\delta_{n}\right)$ for each $i \in N, a\left(\delta_{n}\right)$, and $b\left(\delta_{n}\right)$ converge. From Equation (7) of the characteristic system for the model with time-invariant recognition probabilities with know that $b\left(\delta_{n}\right)-a\left(\delta_{n}\right)=1-\delta_{n}$, so the sequences $a\left(\delta_{n}\right)$ and $b\left(\delta_{n}\right)$ converge to the same limit. Denote this common limit by $z$. Since $a\left(\delta_{n}\right) \leq x_{i}\left(\delta_{n}\right) \leq b\left(\delta_{n}\right)$ the sequence $x_{i}\left(\delta_{n}\right)$ converges to $z$ for each $i \in N$ and so does the sequence $\mu x\left(\delta_{n}\right)$. 
To prove that $z$ is generalized fixed point of the function $1-F$, let $\varepsilon>0$. Then for $n$ large enough

$$
z-\varepsilon \leq a\left(\delta_{n}\right) \leq b\left(\delta_{n}\right) \leq z+\varepsilon .
$$

Because $F$ is a non-decreasing function,

$$
F(z-\varepsilon) \leq F\left(a\left(\delta_{n}\right)\right) \leq F\left(b\left(\delta_{n}\right)\right) \leq F(z+\varepsilon) .
$$

Applying Proposition 5.1, we find that

$$
1-F(z+\varepsilon) \leq 1-F\left(b\left(\delta_{n}\right)\right) \leq \mu x\left(\delta_{n}\right) \leq 1-F\left(a\left(\delta_{n}\right)\right) \leq 1-F(z-\varepsilon) .
$$

Taking the limit we obtain the desired inequality

$$
1-F(z+\varepsilon) \leq z \leq 1-F(z-\varepsilon) .
$$

The result follows because $\varepsilon>0$ is arbitrary.

As we see from Theorem 5.1, in the model with time-invariant recognition probabilities the common social acceptance set collapses to a point as the discount factor approaches one. As a consequence, the limit equilibrium proposals of all players are the same.

Consider the case where all players have the same probability of making a proposal. We take $N$ sufficiently large and preferences sufficiently dispersed, so that for all practical purposes we may assume $F$ is continuous and strictly increasing. The median voter result states that the group of players selects the alternative $z_{\mathrm{m}}$ satisfying $F\left(z_{\mathrm{m}}\right)=1 / 2$. According to Theorem 5.1, the alternative $z_{\mathrm{b}}$ satisfying $z_{\mathrm{b}}+F\left(z_{\mathrm{b}}\right)=1$ is chosen in our limit equilibrium. The alternatives $z_{\mathrm{m}}$ and $z_{\mathrm{b}}$ are the same and equal to $1 / 2$ if and only if $F(1 / 2)=1 / 2$, i.e. the median voter's most preferred outcome is $1 / 2$. If $F(1 / 2)<1 / 2$, then it follows that $z_{\mathrm{m}}>z_{\mathrm{b}} \geq 1 / 2$, and if $F(1 / 2)>1 / 2$, then $z_{\mathrm{m}}<z_{\mathrm{b}} \leq 1 / 2$.

The median voter outcome is more extreme than the outcome selected in our bargaining model. The underlying intuition is that the bargaining model requires unanimous agreement and therefore also the agreement of both the players $i_{0}$ and $i_{1}$. This causes a tendency to select outcomes in the middle. For the median voter model, the votes of half of the players are sufficient, and the opinion of the other players can be safely ignored.

Most striking is the case where the ideal point of each player is either 0 or $1, \mu(\{i \in$ $\left.\left.N \mid p_{i}=0\right\}\right)=(1+\varepsilon) / 2$ and $\mu\left(\left\{i \in N \mid p_{i}=1\right\}\right)=(1-\varepsilon) / 2$ for some $\varepsilon>0$. Then $z_{\mathrm{m}}=0$ and $z_{\mathrm{b}}=(1-\varepsilon) / 2$. When $\varepsilon$ converges to zero, the median voter outcome converges to 0 and the limit equilibrium outcome to $1 / 2$.

With general Markov recognition probabilities, different proposers face different social acceptance sets. While it is still true that a social acceptance set for the proposal of a given proposer collapses to point, this point is in general different for different proposers and Theorem 5.1 does not simply carry over to the general case. 


\subsection{Symmetric recognition probabilities}

In this subsection we assume that $N \subset Z$ and $p_{i}=i$ for all $i \in N$. The player set $N$ is said to be symmetric (around $1 / 2$ ) if $1-i \in N$ whenever $i \in N$. Suppose $N$ is symmetric. The recognition probabilities $\Pi$ are said to be symmetric (around $1 / 2)$ if $\pi(1-i \mid k)=\pi(i \mid k)$ for all $i$ and $k$ in $N$.

Proposition 5.2 Assume $N \subset Z$ and $p_{i}=i$ for all $i \in N$. Assume the player set $N$ and the recognition probabilities $\Pi$ are symmetric. Let $x_{i}^{-}=\delta / 2$ and $x_{i}^{+}=1-\delta / 2$ for all $i \in N$ and let $x$ be given by Equation (8) with $a=\delta / 2$ and $b=1-\delta / 2$. Then $\left(x, x^{-}, x^{+}\right)$is $a$ bargaining equilibrium.

Proof. We verify that the tuple $\left(x, x^{-}, x^{+}\right)$satisfies Equations (5)-(6).

It is clear from Figure 1 that Equation (6) holds. Since $b=1-a$, the function $x$ is symmetric in the sense that $x_{1-i}=1-x_{i}$ for all $i \in N$. Therefore, for any $k \in N$,

$$
\begin{aligned}
2(\Pi x)_{k} & =\sum_{i \in N} \pi(i \mid k) x_{i}+\sum_{i \in N} \pi(1-i \mid k) x_{1-i} \\
& =\sum_{i \in N} \pi(i \mid k) x_{i}+\sum_{i \in N} \pi(i \mid k) x_{1-i} \\
& =\sum_{i \in N} \pi(i \mid k)=1,
\end{aligned}
$$

where the first equality holds because $N$ is symmetric, the second one holds because $\Pi$ is symmetric, and the third one holds because $x$ is symmetric. We see that $\Pi x$ is identically equal to $1 / 2$. It follows that Equation (5) holds.

Thus, when $N$ and $\Pi$ are symmetric, the bargaining equilibrium resembles the one in the case of time-invariant recognition probabilities in that all players face the same social acceptance set. As $\delta$ approaches one, the equilibrium proposals of all players converge to $1 / 2$. Notice however, that, unlike the case of time-invariant recognition probabilities, the expected payoffs $y_{i \mid k}$ and the individual acceptance sets $A_{i \mid k}$ depend on $k$.

\subsection{Deterministic recognition rules}

Let $r: N \rightarrow N$ be a recognition rule. Equation (5) can then be rewritten as

$$
x_{i}^{-}=\delta x_{r(i)} \text {, and } x_{i}^{+}=1-\delta+\delta x_{r(i)} \text { for all } i \in N \text {. }
$$

Claim (a) of Proposition 5.3 below presents a case where any player, once chosen to be the proposer, remains the proposer for the rest of the game. The result is, not surprisingly, that each player proposes his own ideal point. Claim (b) contains the model of Rubinstein (1982) as a special case. It deals with the case where any player $i$, once chosen to be the proposer, alternates with player $1-i$ in being the proposer for the rest of the game. 
Proposition 5.3 Assume $N \subset Z$ and $p_{i}=i$ for all $i \in N$. Consider a game $\Gamma$ with a deterministic recognition rule $r$.

(a) Let $r$ be the identity. The bargaining equilibrium $\left(x, x^{-}, x^{+}\right)$is given by $x_{i}^{-}=\delta i$, $x_{i}^{+}=1-\delta+\delta i$ and $x_{i}=i$ for all $i \in N$.

(b) Suppose the set $N$ is symmetric around $1 / 2$ and $r(i)=1-i$. Let $a=\delta /(1+\delta)$ and $b=1 /(1+\delta)$. Then the bargaining equilibrium $\left(x, x^{-}, x^{+}\right)$is given by

$$
\begin{gathered}
x_{i}= \begin{cases}a, & \text { if } i \leq a, \\
i, & \text { if } a \leq i \leq b, \\
b, & \text { if } b \leq i,\end{cases} \\
x_{i}^{-}=\left\{\begin{array}{ll}
\delta /(1+\delta), & \text { if } i \leq a, \\
\delta-\delta i, & \text { if } a \leq i \leq b, \\
\delta^{2} /(1+\delta), & \text { if } b \leq i,
\end{array} \quad x_{i}^{+}= \begin{cases}\left(1+\delta-\delta^{2}\right) /(1+\delta), & \text { if } i \leq a, \\
1-\delta i, & \text { if } a \leq i \leq b, \\
b, & \text { if } b \leq i .\end{cases} \right.
\end{gathered}
$$

Proof. Claim (a) is obvious. Consider claim (b). Figure 2 illustrates the bargaining equilibrium. It is clear from the figure that $x_{i}$ is the point of $\left[x_{i}^{-}, x_{i}^{+}\right]$closest to $i$, so that Equation (6) holds. Notice that because $b=1-a, x$ is symmetric in the sense that $x_{1-i}=1-x_{i}$ for all $i \in N$. Using this property, it is straightforward to verify that Equation (9) holds as well.

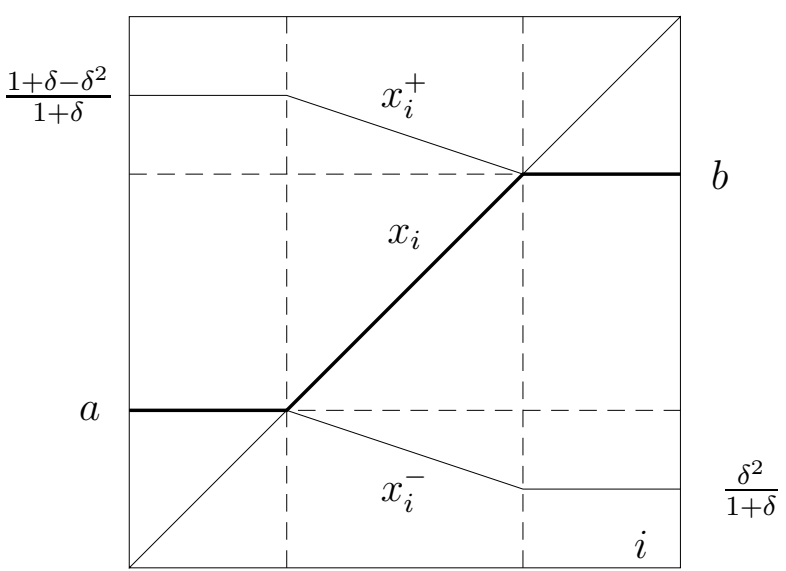

Figure 2: Illustration of $x, x^{-}$and $x^{+}$as in claim (b) of Proposition 5.3.

In part (a) of Proposition 5.3, the equilibrium proposals do not depend on $\delta$.

Figure 2 illustrates the bargaining equilibrium in part (b) of Proposition 5.3. Observe that both $x_{i}^{-}$and $x_{i}^{+}$are non-increasing functions of $i$. The equilibrium proposal $x_{i}$ is nondecreasing in $i$. The equilibrium proposal of each player converges to $1 / 2$ as $\delta$ approaches one. 


\section{Two Results on Stochastic Matrices}

This section provides the mathematical results needed to analyze the asymptotic behavior of bargaining equilibria. Given a set $S \subset N$ let $S^{\text {c }}$ denote the set $N \backslash S$. Let $\pi(S \mid i)=$ $\sum_{j \in S} \pi(j \mid i)$.

\subsection{Absorbing sets and transient states}

The state $j$ is said to lead to state $i$, written as $j \rightarrow i$, if $i=j$ or if there exists a natural number $n$ such that $\pi^{n}(i \mid j)>0$. The states $j$ and $i$ communicate, written as $j \leftrightarrow i$, if $j \rightarrow i$ and $i \rightarrow j$. The relation $\leftrightarrow$ is an equivalence relation. An equivalence class $S$ of the relation $\leftrightarrow$ is called an absorbing set if $\pi(S \mid i)=1$ for all $i \in S$. A state $i$ is said to be transient if it is not an element of any absorbing set. We let $\mathcal{A}$ be the collection of absorbing sets and $D$ be the set of transient states. Figure 3 illustrates.

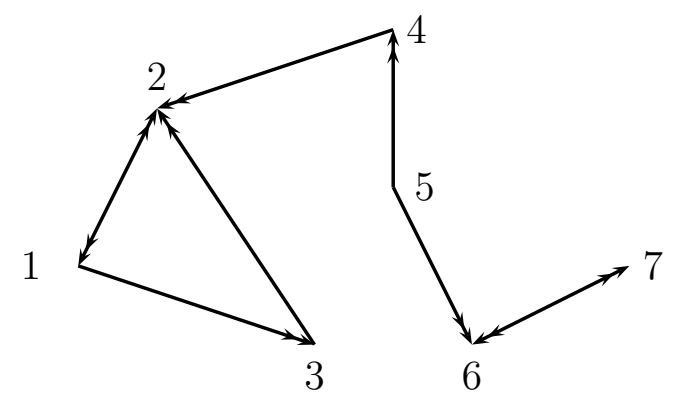

Figure 3: Transition probabilities.

There is a double arrow from $j$ to $i$ if the one-step probability $\pi(i \mid j)$ of transition from $j$ to $i$ is non-zero. The depicted Markov process has two absorbing sets, $\{1,2,3\}$ and $\{6,7\}$. The states 4 and 5 are transient states. Starting from state 5 there is a non-zero probability to enter either absorbing set, while starting from the state 4 the process enters the absorbing set $\{1,2,3\}$ with probability 1.

Given a subset $S$ of $N$ let $\Pi_{S}$ denote the restriction of $\Pi$ to the states in $S$. Notice that if $S$ is an absorbing set, then $\Pi_{S}$ is a stochastic matrix, i.e. the the elements in each row of $\Pi_{S}$ add up to 1 . A stochastic matrix $\Pi$ is said to be irreducible if $N$ is the only absorbing set. If $S$ is an absorbing set, then the stochastic matrix $\Pi_{S}$ is irreducible.

Given an absorbing set $S$, an element $\mu_{S}$ of $\mathbb{R}^{S}$ is said to be a stationary distribution on $S$ if $\mu_{S} \Pi_{S}=\mu_{S}$ and $\sum_{i \in S} \mu_{S}(i)=1$. By convention, $\mu_{S}$ is a row vector. For each absorbing set $S$ there exists a unique stationary distribution on $S$, see Theorem 4.1 in Seneta (2006). It will be convenient to extend $\mu_{S}$ to an element of $\mathbb{R}^{N}$ by letting $\mu_{S}(i)=0$ for all $i \in S^{\mathrm{c}}$. 


\subsection{The convergence of the matrix $\Psi(\delta)$}

Given $\delta \in[0,1)$, define the matrix $\Psi=\Psi(\delta)$ as follows:

$$
\Psi=(1-\delta) \sum_{n=0}^{\infty} \delta^{n} \Pi^{n}=(1-\delta)(I-\delta \Pi)^{-1} .
$$

The following is a well-known result.

Theorem 6.1 Let $\Pi$ be a stochastic irreducible matrix. Let $\mu_{N}$ denote the unique stationary distribution on $N$. As $\delta$ approaches one from below, each row of the matrix $\Psi(\delta)$ converges to $\mu_{N}$.

We shall use the following straightforward corollary of Theorem 6.1.

Corollary 6.1 Let $S$ be an absorbing set and let $\mu_{S}$ be the corresponding stationary distribution on $S$. As $\delta$ approaches one from below, each row of the matrix $\Psi(\delta)$ corresponding to a state in $S$ converges to $\mu_{S}$.

Proof. Define $\Psi_{S}(\delta)$ by the equation

$$
\Psi_{S}(\delta)=(1-\delta) \sum_{n=0}^{\infty} \delta^{n} \Pi_{S}^{n} .
$$

Since $S$ is an absorbing set, the matrix $\Pi_{S}$ is irreducible, and Theorem 6.1 applies to show that each row of the matrix $\Psi_{S}(\delta)$ converges to $\mu_{S}$. Now, the elements of the matrix $\Pi$ are related to the elements in $\Pi_{S}$ by the equation

$$
\pi^{n}(i \mid j)= \begin{cases}\pi_{S}^{n}(i \mid j), & \text { if }(i, j) \in S \times S \\ 0, & \text { if }(i, j) \in S^{c} \times S .\end{cases}
$$

Thus the row $j \in S$ of the matrix $\Pi^{n}$ equals the row $j$ of the matrix $\Pi_{S}^{n}$ extended by the zeros corresponding to states in $S^{\mathrm{c}}$. It follows that row $j \in S$ of $\Psi(\delta)$ equals row $j$ of $\Psi_{S}(\delta)$ extended by zeros corresponding to states in $S^{c}$. The result follows.

\subsection{The Perron-Frobenius Theorem and absorption probabilities}

We state a version of Perron-Frobenius Theorem for a stochastic irreducible matrix that will be sufficient for our purposes (see Theorem 1.5 in Seneta (2006)).

Theorem 6.2 Let $\Pi$ be a stochastic irreducible matrix. An element $x$ in $\mathbb{R}^{N}$ satisfies the equation $\Pi x=x$ if and only if it is constant on $N$. 
Using Theorem 6.2 we obtain a characterization of the eigenspace of a general stochastic matrix associated with an eigenvalue one.

Starting from any transient state $i \in D$, the Markov process eventually leaves $D$ and enters one of the absorbing sets where it stays forever. Given an absorbing set $S$ and a transient state $i$, let $\varphi(S \mid i)$ denote the probability for the process to enter the set $S$ given the initial state $i$. Let $\varphi(S \mid D)$ be the column vector of absorption probabilities $\varphi(S \mid i)$ as the index $i$ ranges over all transient states. Let $\pi(S \mid D)$ be the column vector of probabilities $\pi(S \mid i)$ as the index $i$ ranges over all transient states. The result quoted below combines Theorems 4.3 and 4.4 from Seneta (2006).

Theorem 6.3 Let $\Pi$ be a stochastic matrix. Then the matrix $I_{D}-\Pi_{D}$ is invertible. Furthermore, $\varphi(S \mid D)=\left[I_{D}-\Pi_{D}\right]^{-1} \pi(S \mid D)$ for each absorbing set $S$.

The following corollary provides a characterization of the eigenspace of a general stochastic matrix $\Pi$ associated with an eigenvalue one.

Corollary 6.2 Let $\Pi$ be a stochastic matrix. An element $x$ of $\mathbb{R}^{N}$ satisfies the equation $\Pi x=x$ if and only if (a) the vector $x$ is constant on each absorbing set, and (b) for each state $i \in D$

$$
x_{i}=\sum_{S \in \mathcal{A}} \varphi(S \mid i) x_{S}
$$

where $x_{S}$ is the value of $x$ on an absorbing set $S$.

Proof. Suppose $\Pi x=x$.

Let $S$ be an absorbing set and let $x_{\mid S}$ denote the restriction of $x$ to $S$. It follows that $\Pi_{S} x_{\mid S}=x_{\mid S}$. Since $S$ is an absorbing set, the matrix $\Pi_{S}$ is a stochastic irreducible matrix. By Theorem 6.2, the vector $x_{\mid S}$ is constant on the set $S$.

Let $x_{S}$ denote the value of $x$ on $S$ and $x_{\mid D}$ denote the restriction of $x$ to $D$. Then the equations corresponding the transient states can be written as

$$
\sum_{S \in \mathcal{A}} \pi(S \mid D) x_{S}+\Pi_{D} x_{\mid D}=x_{\mid D}
$$

Solving the system, we obtain

$$
x_{\mid D}=\left[I_{D}-\Pi_{D}\right]^{-1} \sum_{S \in \mathcal{A}} \pi(S \mid D) x_{S}=\sum_{S \in \mathcal{A}} \varphi(S \mid D) x_{S} .
$$

The result follows. The converse direction is now easy to prove.

Consider a Markov process as in Figure 3. If the vector $x$ satisfies the equation $\Pi x=x$, then $x_{1}=x_{2}=x_{3}$ and $x_{6}=x_{7}$, since $\{1,2,3\}$ and $\{6,7\}$ are the absorbing sets of the process. Furthermore, $x_{4}=x_{1}$, because, starting from state 4 , the process enters the absorbing set $\{1,2,3\}$ with probability 1 . Finally, $x_{5}$ is a convex combination of $x_{1}$ and $x_{6}$ with the weights equal to the respective absorbtion probabilities. 


\section{The Limit Equilibrium}

In this section we study the limit of the bargaining equilibrium when the discount factor tends to one from below. The limit is shown to exist and is called the limit equilibrium. We characterize the limit equilibrium as a generalized fixed point of a particular function. We study under what conditions all players make the same proposal in the limit equilibrium.

Theorem 7.1 below is the main result of the paper. The proof of the theorem relies on Proposition 7.1 below and Corollaries 6.1 and 6.2 in the Appendix.

Given an $x \in Z^{N}$ let $B(x)=\left\{i \in N \mid x_{i}<p_{i}\right\}$. Notice that $B(x) \supset B(\dot{x})$ whenever $x \leq \dot{x}$.

Proposition 7.1 Let $\left(x, x^{-}, x^{+}\right)$be a bargaining equilibrium. Then $\Psi 1_{B^{-}} \leq x \leq \Psi 1_{B^{+}}$ where $B^{-}=B\left(x^{+}\right)$and $B^{+}=B\left(x^{-}\right)$.

Proof. Define the elements $z^{-}$and $z^{+}$of $Z^{N}$ as follows:

$$
z_{k}^{-}=\left\{\begin{array}{ll}
x_{k}^{-}, & \text {if } p_{k} \leq x_{k}^{+}, \\
x_{k}^{+}, & \text {otherwise },
\end{array} \quad \text { and } \quad z_{k}^{+}= \begin{cases}x_{k}^{-}, & \text {if } p_{k} \leq x_{k}^{-} \\
x_{k}^{+}, & \text {otherwise }\end{cases}\right.
$$

Using Equation (6) of the characteristic system, it is easy to see that $z^{-} \leq x \leq z^{+}$. Since $\Pi$ preserves the relation $\leq$, we also have $\Pi z^{-} \leq \Pi x \leq \Pi z^{+}$, and, for each natural $n$

$$
\Pi^{n} z^{-} \leq \Pi^{n} x \leq \Pi^{n} z^{+} .
$$

Using Equation (5) of the characteristic system, we can rewrite $z^{-}$and $z^{+}$as

$$
z^{-}=\delta \Pi x+(1-\delta) 1_{B^{-}} \text {and } z^{+}=\delta \Pi x+(1-\delta) 1_{B^{+}} .
$$

Applying $\Pi^{n}$ to each side of the equations, we obtain

$$
\Pi^{n} z^{-}=\delta \Pi^{n+1} x+(1-\delta) \Pi^{n} 1_{B^{-}} \text {and } \Pi^{n} z^{+}=\delta \Pi^{n+1} x+(1-\delta) \Pi^{n} 1_{B^{+}} .
$$

Now we have the following chain of inequalities:

$$
\begin{aligned}
x & \leq z^{+}=\delta \Pi x+(1-\delta) 1_{B^{+}} \\
& \leq \delta \Pi z^{+}+(1-\delta) 1_{B^{+}}=\delta^{2} \Pi^{2} x+(1-\delta) 1_{B^{+}}+(1-\delta) \delta \Pi 1_{B^{+}} \\
& \leq \cdots \\
& \leq \delta^{n+1} \Pi^{n+1} x+(1-\delta) \sum_{i=0}^{n} \delta^{n} \Pi^{n} 1_{B^{+}} .
\end{aligned}
$$

As $n$ goes to infinity, the first term of the last expression vanishes, while the second term converges to $\Psi 1_{B^{+}}$. We thus obtain the upper bound $x \leq \Psi 1_{B^{+}}$on $x$. To obtain the lower bound, consider the chain of inequalities

$$
\begin{aligned}
x & \geq z^{-}=\delta \Pi x+(1-\delta) 1_{B^{-}} \\
& \geq \delta \Pi z^{-}+(1-\delta) 1_{B^{-}}=\delta^{2} \Pi^{2} x+(1-\delta) 1_{B^{-}}+(1-\delta) \delta \Pi 1_{B^{-}} \\
& \geq \cdots \\
& \geq \delta^{n+1} \Pi^{n+1} x+(1-\delta) \sum_{i=0}^{n} \delta^{n} \Pi^{n} 1_{B^{-}} .
\end{aligned}
$$


Taking the limit as $n$ goes to infinity, we obtain the lower bound $x \geq \Psi 1_{B^{-}}$.

For each absorbing set $S$ define $F_{S}: \mathbb{R} \rightarrow Z$ by letting $F_{S}(z)=\mu_{S}\left(\left\{i \in S \mid p_{i} \leq z\right\}\right)$. As $F_{S}$ is a non-decreasing function, the function $1-F_{S}$ has a unique generalized fixed point.

Definition 7.1 The limit equilibrium is an element of $Z^{N}$, denoted $x^{\ell}$, satisfying the following conditions: (A) The vector $x^{\ell}$ is constant on each absorbing set $S$, (b) the value $x_{S}^{\ell}$ of $x^{\ell}$ on $S$ is the generalized fixed point of the function $1-F_{S}$, and (C) the value of $x^{\ell}$ on each transient state $i$ is given by

$$
x_{i}^{\ell}=\sum_{S \in \mathcal{A}} \varphi(S \mid i) x_{S}^{\ell}
$$

The conditions (A) and (C) of Definition 7.1 are equivalent to the requirement that $\Pi x^{\ell}=$ $x^{\ell}$, see Corollary 6.2.

Given a discount factor $\delta \in[0,1)$, we let $\left(x(\delta), x^{-}(\delta), x^{+}(\delta)\right)$ denote the bargaining equilibrium.

Theorem 7.1 The limits $\lim _{\delta \uparrow 1} x(\delta), \lim _{\delta \uparrow 1} x^{-}(\delta)$, and $\lim _{\delta \uparrow 1} x^{+}(\delta)$ exist. All three limits are equal to $x^{\ell}$.

Proof. Let $\delta_{n}$ be a sequence in $[0,1)$ converging to 1 . We must show that the sequences $x\left(\delta_{n}\right), x^{-}\left(\delta_{n}\right)$, and $x^{+}\left(\delta_{n}\right)$ converge to $x^{\ell}$. Without loss of generality assume that the sequence $x\left(\delta_{n}\right)$ converges to an element to $x$ of $Z^{N}$.

The sequence $\Pi x\left(\delta_{n}\right)$ converges $\Pi x$. By Equation (5) of the characteristic system, $x^{-}\left(\delta_{n}\right)=\delta_{n} \Pi x\left(\delta_{n}\right)$ and $x^{+}\left(\delta_{n}\right)=\left(1-\delta_{n}\right) 1_{N}+\delta_{n} \Pi x\left(\delta_{n}\right)$. It follows that the sequences $x^{-}\left(\delta_{n}\right)$ and $x^{+}\left(\delta_{n}\right)$ converge to $\Pi x$. Since $x^{-}\left(\delta_{n}\right) \leq x\left(\delta_{n}\right) \leq x^{+}\left(\delta_{n}\right)$, it then follows that $x\left(\delta_{n}\right)$ converges to $\Pi x$. But we know that $x\left(\delta_{n}\right)$ converges to $x$. Thus $x=\Pi x$.

We conclude that $x$ is the eigenvector of the matrix $\Pi$ associated with the eigenvalue one. We now use Corollary 6.2 in Section 6 that provides a complete description of the eigenspace of the matrix $\Pi$ associated with eigenvalue one. Thus Corollary 6.2 implies that $x$ is constant on each absorbing set and that Equation (10) holds. It remains to be shown that the value $x_{S}$ of $x$ on each absorbing set $S$ is a generalized fixed point of the function $1-F_{S}$.

We already know that the sequences $x^{-}\left(\delta_{n}\right)$ and $x^{+}\left(\delta_{n}\right)$ converge to $x$. Since $N$ is a finite set, for $n$ large enough the following inequalities hold:

$$
x-1_{N} \varepsilon \leq x^{-}\left(\delta_{n}\right) \leq x^{+}\left(\delta_{n}\right) \leq x+1_{N} \varepsilon .
$$

It follows that for $n$ large enough we have the inclusions

$$
B\left(x+1_{N} \varepsilon\right) \subset B\left(x^{+}\left(\delta_{n}\right)\right) \subset B\left(x^{-}\left(\delta_{n}\right)\right) \subset B\left(x-1_{N} \varepsilon\right) .
$$

These inclusions imply the inequalities

$$
1_{B\left(x+1_{N} \varepsilon\right)} \leq 1_{B\left(x^{+}\left(\delta_{n}\right)\right)} \leq 1_{B\left(x^{-}\left(\delta_{n}\right)\right)} \leq 1_{B\left(x-1_{N} \varepsilon\right)} .
$$


We then also have the inequalities

$$
\Psi\left(\delta_{n}\right) 1_{B\left(x+1_{N} \varepsilon\right)} \leq \Psi\left(\delta_{n}\right) 1_{B\left(x^{+}\left(\delta_{n}\right)\right)} \leq x\left(\delta_{n}\right) \leq \Psi\left(\delta_{n}\right) 1_{B\left(x^{-}\left(\delta_{n}\right)\right)} \leq \Psi\left(\delta_{n}\right) 1_{B\left(x-1_{N} \varepsilon\right)},
$$

where the middle inequalities follow from Proposition 7.1.

Now let $S$ be an absorbing set and let $x_{S}$ be the value of $x$ on $S$. Let $k \in S$ and consider the inequality

$$
\left[\Psi\left(\delta_{n}\right) 1_{B\left(x+1_{N} \varepsilon\right)}\right]_{k} \leq x_{k}\left(\delta_{n}\right) \leq\left[\Psi\left(\delta_{n}\right) 1_{B\left(x-1_{N} \varepsilon\right)}\right]_{k} .
$$

By Corollary 6.1 in Section 6 , row $k$ of the matrix $\Psi\left(\delta_{n}\right)$ converges to $\mu_{S}$ as $n$ goes to infinity. We thus obtain the inequalities

$$
\mu_{S} 1_{B\left(x+1_{N} \varepsilon\right)} \leq x_{S} \leq \mu_{S} 1_{B\left(x-1_{N} \varepsilon\right)} .
$$

Now, for any set $B \subset N$ we have $\mu_{S} 1_{B}=\mu_{S}(B \cap S)$. Furthermore, $B\left(x+1_{N} \varepsilon\right) \cap S=\{i \in$ $\left.S \mid x_{S}+\varepsilon<p_{i}\right\}$ and $B\left(x-1_{N} \varepsilon\right) \cap S=\left\{i \in S \mid x_{S}-\varepsilon<p_{i}\right\}$. Thus, the preceding inequality can be rewritten as

$$
\mu_{S}\left(\left\{i \in S \mid x_{S}+\varepsilon<p_{i}\right\}\right) \leq x_{S} \leq \mu_{S}\left(\left\{i \in S \mid x_{S}-\varepsilon<p_{i}\right\}\right) .
$$

The left-hand side equals $1-F_{S}\left(x_{S}+\varepsilon\right)$ and the right-hand side equals $1-F_{S}\left(x_{S}-\varepsilon\right)$. Thus we can once again rewrite the preceding inequality as

$$
1-F_{S}\left(x_{S}+\varepsilon\right) \leq x_{S} \leq 1-F_{S}\left(x_{S}-\varepsilon\right) .
$$

Since $\varepsilon>0$ is arbitrary, it follows that $x_{S}$ is the generalized fixed point of the function $1-F_{S}$, as desired.

Now we identify some special cases where $x^{\ell}$ is constant on the entire player set $N$. In these cases every player makes the same proposal in the limit equilibrium.

Proposition 7.2 If the proposer selection process has a unique absorbing set, then $x^{\ell}$ is constant on $N$.

Proof. The result follows directly from the definition of limit equilibrium. The vector $x^{\ell}$ is constant on the absorbing set $S$. Furthermore, starting from each state $i$ outside $S$ the process is absorbed into $S$ with probability 1 , that is $\varphi(S \mid i)=1$.

Proposition 7.3 If the matrix $\Pi$ is irreducible, then $x^{\ell}$ is constant on $N$.

Proof. If the matrix $\Pi$ is irreducible, then $N$ is the only absorbing set.

Proposition 7.4 Suppose the proposer selection process has time-invariant recognition probabilities $\mu$. Then $x^{\ell}$ is constant on $N$ and its value is the generalized fixed point of the function $1-F$, where $F(z)=\mu\left(\left\{i \in N \mid p_{i} \leq z\right\}\right)$.

Proof. If $\mu$ are the time-invariant recognition probabilities, then $S=\{i \in N \mid \mu(i)>0\}$ is the only absorbing set, and the stationary distribution corresponding to $S$ is $\mu$. The result now follows from Proposition 7.2 and the definition of $x^{\ell}$. 


\section{Conclusion}

We have formulated the important problem of how to select an outcome in a one-dimensional set of alternatives as a non-cooperative bargaining problem. We consider the case where a proposal is only accepted if all players approve of it. Subgame perfect equilibrium in stationary strategies is unique up to unimportant details of individual acceptance sets and is called bargaining equilibrium. We provide a simple characterization of the bargaining equilibrium.

In our model, proposers are selected according to a general Markov process. This captures two cases that feature prominently in the literature: the one with time-invariant recognition probabilities and the one with a deterministic proposer selection rule. Our characterization of bargaining equilibrium simplifies further in these important special cases.

Finally, we study the limit equilibrium, the limit of a sequence of bargaining equilibria as the discount factor tends to one. We give an explicit description of the limit equilibrium as the generalized fixed point of a function that is intimately related to the time-invariant distribution of the proposer selection process. Surprisingly, all players make the same proposal in the limit equilibrium under a wide variety of specifications of the proposer selection process.

We may compare the alternative selected in our bargaining model to the one selected according to the median voter theorem. We find that the bargaining alternative is less extreme, i.e. closer to the middle, than the most preferred alternative by the median voter. The bargaining alternative $z$ is the one that satisfies $z+F(z)=1$, with $F$ a distribution function related to the players' most preferred points. The median voter theorem specifies $z$ such that $F(z)=1 / 2$ as the solution. We have therefore presented a rigorous noncooperative analysis of the choice of an alternative by a group of players that leads to clear predictions and testable implications.

\section{References}

Banks, J.S., And J. Duggan (2000), "A Bargaining Model of Collective Choice," American Political Science Review, 94, 73-88.

Baron, D.P., And J.A. Ferejohn (1989), "Bargaining in Legislatures," American Political Science Review, 83, 1181-1206.

Black, D. (1958), The Theory of Committees and Elections, Cambridge University Press, London.

Cardona, D., And C. Ponsatí (2007), "Bargaining One-Dimensional Social Choices," Journal of Economic Theory, forthcoming. doi: 10.1016/j.jet.2006.12.001.

Cho, S.-J., And J. Duggan (2003), "Uniqueness of Stationary Equilibria in a OneDimensional Model of Bargaining," Journal of Economic Theory, 113, 118-130. 
Eraslan, H. (2002), "Uniqueness of Stationary Equilibrium Payoffs in the Baron-Ferejohn Model," Journal of Economic Theory, 103, 11-30.

Eraslan, H., And A. Merlo (2002), "Majority Rule in a Stochastic Model of Bargaining," Journal of Economic Theory, 103, 31-48.

Haller, H. (1986), "Non-cooperative Bargaining of $N \geq 3$ Players," Economic Letters, 22, 11-13.

Herings, P.J.J., And A. Predtetchinski (2007), Sequential Share Bargaining, METEOR Research Memorandum 07/005, Maastricht University, The Netherlands.

Herrero, M. (1985), A Strategic Bargaining Approach to Market Institutions, PhD Thesis, London School of Economics, London.

Kalandrakis, T. (2006a), "Proposal Rights and Political Power," American Journal of Political Science, 50, 441-448.

Kalandrakis, T. (2006b), "Regularity of Pure Strategy Equilibrium Points in a Class of Bargaining Games," Economic Theory, 28, 309-329.

Knight, B. (2005), "Estimating the Value of Proposal Power," American Economic Review, 95, 1639-1652.

Merlo, A., And C. Wilson (1995), "A Stochastic Model of Sequential Bargaining with Complete Information," Econometrica, 63, 371-399.

Merlo, A., And C. Wilson (1998), "Efficient Delays in a Stochastic Model of Bargaining," Economic Theory, 11, 39-55.

Romer, Th., And H. Rosenthal (1978), "Political Resource Allocation, Controlled Agendas, and the Status Quo," Public Choice, 33, 27-44.

Rubinstein, A. (1982), "Perfect Equilibrium in a Bargaining Model," Econometrica, 50, 97-109.

Seneta, E. (2006), Non-negative Matrices and Markov Chains, Series in Statistics, SpringerVerlag, Berlin. 